\title{
Ultrasound enhanced antitumor activity of liposomal doxorubicin in mice
}

\author{
Eirik Hagtvet ${ }^{1}$, Tove J. Evjen ${ }^{2,3}$, Dag Rune Olsen ${ }^{4}$, Sigrid L. Fossheim ${ }^{2,5}$, and Esben A. Nilssen ${ }^{2}$ \\ ${ }^{1}$ Department of Radiation Biology, Institute for Cancer Research, Oslo University Hospital and University of Oslo, \\ Oslo, Norway, ${ }^{2}$ Epitarget AS, Oslo, Norway, ${ }^{3}$ Faculty of Health Sciences, Department of Pharmacy, Drug Transport and \\ Delivery Group, University of Tromsø, Tromsø, Norway, ${ }^{4}$ Faculty of Mathematics and Natural Sciences, University of \\ Bergen, Bergen, Norway, and ${ }^{5}$ Present address: Clavis Pharma ASA, Oslo, Norway
}

\begin{abstract}
Liposomal encapsulation of doxorubicin (DXR) improves tumor accumulation and reduces adverse effects. One possible strategy for further optimization of this delivery technology would be to design the liposome carrier to release its content within the tumor tissue in response to specific stimuli such as ultrasound (US). In this study, the tumor uptake properties and therapeutic efficacy of 1,2 distearoyl-sn-glycero-3-phosphatidylethanolamine-based liposomes containing DXR were investigated in nude mice bearing tumor xenografts. The liposomal DXR formulation alone showed no inhibitory effect on tumor growth. However, upon exposure to low frequency US in situ inhibition of tumor growth was demonstrated.
\end{abstract}

Keywords: Therapeutic effect, low frequency ultrasound, drug delivery, drug release

\section{Introduction}

Solid tumors represent $85 \%$ of human cancers and most chemotherapeutics used to treat such cases are small molecular weight molecules (Jang et al., 2003). Such molecules distribute non-specifically to both healthy and tumor tissue resulting in therapy-limiting toxicities. One strategy to improve the therapeutic-to-toxicity ratio has been to encapsulate cancer drugs into nanosized liposomes, which accumulate in tumors due to the so-called enhanced permeability and retention effect (Maeda et al., 2000). Here, leaky tumor vessels allow liposomes to extravasate into tumor tissue, while reduced lymphatic tumor drainage results in liposomal accumulation. To avoid excessive liposomal uptake by the mononuclear phagocyte system (MPS), introducing polyethylene glycol (PEG) into the liposomal membrane reduces undesired MPS accumulation and promotes prolonged circulation time (Gabizon et al., 2003). The pegylated liposomal doxorubicin (PL-DXR) formulation, Caelyx ${ }^{\circledR}$, is today in routine clinical use for treatment of various solid tumors (Gabizon et al., 2003). By enclosing DXR in liposomes,
DXR accumulation in the heart is reduced, resulting in less cardiac toxicities compared to conventional DXR (Ewer et al., 2004; Batist, 2007).

The efficacy of liposomal drug delivery to tumor tissue is determined by the blood circulation time, tumor extravasation and the ability of the drug carrier to rapidly release its drug-load upon tumor accumulation. Combining long circulation time and efficient drug release is, however, nontrivial and still constitutes a key challenge within liposomology (Drummond et al., 1999). Several approaches have been proposed to induce drug release from liposomes including hyperthermia(Needham and Dewhirst, 2001), enzymatic- (Davidsen et al., 2001) and pH- (Sudimack et al., 2002) mediated strategies. However, a growing line of evidence suggests that ultrasound (US) may enhance liposomal drug release improving therapeutic effect (Pitt et al., 2004; Schroeder et al., 2007, 2009a).

Earlier animal studies have suggested improved antitumor activity of liposomal cytostatics when combined with low frequency US (LFUS). A recent study indicated

Address for Correspondence: Dag Rune Olsen, Faculty of Mathematics and Natural Sciences, University of Bergen, PO Box 7803, Bergen N-5020, Norway. E-mail: dag.olsen@mnfa.uib.no 
an improved antitumor effect of PL-DXR when combined with LFUS (Myhr and Moan, 2006). Schroeder et al. (2009a) have convincingly shown that combining liposomal cisplatin and LFUS produce an enhanced therapeutic effect compared to liposomal cisplatin alone. However, the observed therapeutic benefit of this approach has so far been marginal.

In the studies mentioned above, the liposomes were not specifically designed for US-mediated drug release. We have previously reported that in vitro sonosensitivity is significantly enhanced for liposomes comprising distearoylphosphatidylethanolamine (DSPE) compared to phosphatidylcholine-based liposomes (Evjen et al., 2010). The positive correlation between DSPE and sonosensitivity was suggested to be related to the non-bilayer forming characteristics of the lipid, leading to membrane perturbations on US exposure and concomitant drug release (Evjen et al., 2010). In this study, the pharmacokinetics and biodistribution of DSPE-based liposomal DXR were investigated in a prostate xenograft model in nude mice, as well as the therapeutic effect of combining the current liposomes with LFUS.

\section{Materials and methods}

\section{Materials}

DXR hydrochloride, provided as lyophilized powder, was supplied from Nycomed, Asker, Norway. The PL-DXR product Caelyx $^{\circledR}$ was supplied by the pharmacy at the Norwegian Radium Hospital, Oslo, Norway (European distributor Schering-Plough). 1,2-Distearoyl-snglycero-3-phosphatidylethanolamine (DSPE), 1,2-distearoyl-sn-glycero-3-phosphatidylcholine (DSPC) and 1,2-distearoyl-sn-glycero-3-phosphatidylethanolamine$N$-(methoxy(PEG)-2000) (DSPE-PEG 2000) were purchased from Genzyme Pharmaceuticals, Liestal, Switzerland. Cholesterol, 4-(2-hydroxyethyl)-1piperazineethanesulfonic acid (HEPES), ammonium sulfate, Triton $\mathrm{X}-100^{\circledR}$ solution and sucrose were obtained from Sigma-Aldrich, Oslo, Norway. Serum of fetal bovine origin was purchased from Autonorm, Sero, Billingstad, Norway.

For anesthesia of mice, a mixture of $2.4 \mathrm{mg} / \mathrm{ml}$ tiletamine and $2.4 \mathrm{mg} / \mathrm{ml}$ zolazepam $\left(\right.$ Zoletil $^{\circledR}$ vet; Virbac Laboratories, Carros, France), $3.8 \mathrm{mg} / \mathrm{ml}$ xylazine (Narcoxyl ${ }^{\circledR}$ vet; Roche, Basel, Switzerland) and $0.1 \mathrm{mg} / \mathrm{ml}$ butorphanol (Torbugesic ${ }^{\circledR}$; Fort Dodge Laboratories, Fort Dodge, IA) was prepared and used. Physiological saline was supplied by Fresenius Kabi, Halden, Norway.

\section{Liposome preparation}

Liposomes composed of DSPE, DSPC, DSPE-PEG 2000 and cholesterol (54.5:10:5.5:30 mole\%) with a mean intensity weighted diameter of $85 \pm 5 \mathrm{~nm}$ were prepared by the thin film hydration and sequential extrusion technique, as previously described by Evjen and coworkers (2010). In brief, lipids were dissolved in a chloroform/methanol mixture $(9 / 1 \mathrm{v} / \mathrm{v})$ at $60^{\circ} \mathrm{C}$, and rotary evaporated to dryness under high vacuum. The resulting dry lipid film was hydrated with $300 \mathrm{mM}$ ammonium sulfate followed by three freeze-thawing cycles and stepwise extrusion (Lipex extruder; Biomembrane Inc., Vancouver, BC, Canada) through Nucleopore polycarbonate filters of decreasing pore size (800 to $80 \mathrm{~nm}$ ) (Nucleopore, West Chester, PA). The lipid hydration, liposome extrusion and thawing process were performed at $75^{\circ} \mathrm{C}$, above the gelto-liquid crystalline phase transition temperature of the phospholipids.

Formation of an ammonium sulfate gradient was achieved by extensive dialysis of extruded liposomes against a $255 \mathrm{mM}$ sucrose solution, with intermediate exchange of the dialysis solution for $\sim 48 \mathrm{~h}$. DXR was added as solution to the liposome dispersion to give a drug-to-lipid ratio of $1: 16 \mathrm{~m} / \mathrm{m}$. The final lipid concentration was $16 \mathrm{mg} / \mathrm{ml}$. To obtain optimal drug entrapment efficiency, the liposome dispersions were, after DXR addition, further incubated for $30 \mathrm{~min}$ under stirring at $75^{\circ} \mathrm{C}$. Any remaining free DXR was removed by liposome dialysis against an isotonic sucrose solution containing 10 mM HEPES ( $\mathrm{pH} 7.4$ ). The final liposomal DXR concentration was quantified by fluorescence measurements as described by Evjen and coworkers (2010).

\section{In vitro liposomal characterization}

The mean intensity-weighted hydrodynamic liposome diameter was determined by photon correlation spectroscopy at $23^{\circ} \mathrm{C}$ and at a scattering angle of $90^{\circ}$ (Nanosizer; Malvern Instruments, Malvern, UK). As an indicator for the width of the particle size distribution, the polydispersity index (PI) was given. Each liposome batch was diluted $1: 200(\mathrm{v} / \mathrm{v})$ with $0.22 \mu \mathrm{m}$ filtered isotonic sucrose solution containing $10 \mathrm{mM}$ HEPES ( $\mathrm{pH}$ 7.4). Triplicate measurements were performed. $\zeta$ Potential measurements were performed using a Malvern Zetasizer 2000 (Malvern Instruments, Malvern, UK). Liposome dispersions were diluted 1:30 (v/v) with $0.22 \mu \mathrm{m}$ filtered isotonic sucrose solution containing $10 \mathrm{mM}$ HEPES ( $\mathrm{pH}$ 7.4). The diluted liposome dispersions were injected into the measuring cell immediately after $\mathrm{pH}$ measurements. Triplicate measurements were performed.

US experiments were performed using a $40 \mathrm{kHz}$ nonfocused $19 \mathrm{~mm}$ diameter US transducer (model VC 754; Sonic and Materials Inc., Newtown, CT) connected to a custom-built sample chamber as described by Huang and MacDonald (2004). The temperature in the sample chamber was kept below $30^{\circ} \mathrm{C}$ during the US exposure by circulating room tempered water through adjacent chambers. The liposome dispersions were diluted in a $1: 500 \mathrm{v} / \mathrm{v}$ ratio with isosmotic sucrose solution containing $10 \mathrm{mM}$ HEPES ( $\mathrm{pH} 7.4$ ) just before the US experiments. The diluted liposome dispersions were exposed to $40 \mathrm{kHz}$ US at a nominal transducer input of $\sim 12 \mathrm{~W} /$ $\mathrm{cm}^{2}$ in continuous mode. Samples were taken out at 0.5 , $1.0,1.5,2.0,4.0$ and $6.0 \mathrm{~min}$ of US. The following equation was used for quantification of US-mediated DXR release: 
\% DXR release $=\frac{\left(F_{\mathrm{t}}-F_{0}\right)}{\left(F_{\max }-F_{0}\right)} * 100$.

Where $F_{0}$ and $F_{\mathrm{t}}$ are the fluorescence intensities of a given liposome sample before and after a given duration $(t)$ of US, respectively, and $F_{\max }$ is the fluorescence intensity after liposome solubilization with surfactant (Triton X-100) to mimick $100 \%$ DXR release. The diluted liposome samples were solubilized with Triton X-100 at a ratio of 50:1 (v/v). Triplicate US measurements were performed.

Liposome stability was studied using a well-established serum-induced leakage assay mimicking biological conditions (Allen and Cleland, 1980; Bonté and Juliano, 1986; Lasic, 1993). Liposome dispersions were diluted 1:125 (v/v) with an isotonic sucrose solution containing $10 \mathrm{mM}$ HEPES ( $\mathrm{pH} 7.4$ ) and $20 \%$ fetal bovine serum and incubated for $24 \mathrm{~h}$ at $37^{\circ} \mathrm{C}$. Time-dependent DXR leakage was quantified by fluorescence measurements of the liposome samples further diluted 1:4(v/v) with the above mentioned sucrose solution, this according to equation 1. Triplicate samples were measured.

The temperature sensitivity of the liposomes was investigated by diluting liposome dispersions 1:125 (v/v) with isotonic sucrose solution containing $10 \mathrm{mM}$ HEPES ( $\mathrm{pH} 7.4$ ) and exposed to $60^{\circ} \mathrm{C}$ for $2 \mathrm{~h}$. Temperatureinduced DXR leakage was quantified by fluorescence measurements of the liposome samples further diluted as described above, according to equation 1. Triplicate samples were measured.

All fluorescence intensity measurements were performed using a fluorescence spectrometer from Ocean Optics (model QE65000; Duiven, the Netherlands). The excitation and emission wavelength were 488 and $595 \mathrm{~nm}$, respectively.

\section{Experimental animals}

Male athymic nude Balb/c mice were provided by the Department of Comparative Medicine (animal facility), the Norwegian Radium Hospital. CWR22 prostate adenocarcinoma, obtained from surgical specimens (Pretlow et al., 1993), were serially transplanted in mice. In brief, by blunt dissection through a skin incision, a tumor fragment $(\sim 2 \times 2 \times 2 \mathrm{~mm})$ was subcutaneously (s.c.) implanted into the flank of 4-5-week-old mice. The skin incision was sealed with topical skin adhesive and 4 weeks later a tumor xenograft of $5-8 \mathrm{~mm}$ in diameter developed. The mice were housed in transparent boxes with bedding material, fed ad libitum and kept under specific pathogen-free conditions. The temperature and relative humidity were kept constant at $20-21^{\circ} \mathrm{C}$ and $60 \%$, respectively. At the end of the experiments, all animals were euthanized by cervical dislocation. All procedures were performed according to protocols approved by the National Animal Research Authority and carried out in compliance with the European Convention for the Protection of Vertebrates Used for Scientific Purposes.

\section{Pharmacokinetic and biodistribution studies}

Liposomal DXR was administered 4 weeks after tumor transplantation when the mice carried xenografts sized $5-8 \mathrm{~mm}$ in diameter. Animals received $3.5 \mathrm{mg} / \mathrm{kg}$ liposomal DXR as a single intravenous (i.v.) bolus through the tail vein under anesthesia induced by s.c. administration of $0.1 \mathrm{ml}$ anesthetic agent. Animals were sacrificed in groups of four at different postinjection time points ( 1 , $3,8,12,18,24,30,48 \mathrm{~h})$. Tumor, liver, spleen and kidneys were excised and their weight registered. The total blood volume was collected by cardiac puncture using heparinized syringes and stored in heparinized tubes. All tissue and blood samples were kept on ice bath until storage at $-80^{\circ} \mathrm{C}$.

\section{Quantification of DXR in blood and tissues}

Quantification of DXR was performed similarly to the one described by Gabizon et al. (1989). In brief, $0.1 \mathrm{ml}$ whole blood samples (lysed due to freezing) were mixed with $1.9 \mathrm{ml}$ acidified ethanol $(0.3 \mathrm{M} \mathrm{HCl}$ in $50 \%$ ethanol, $\mathrm{pH}<1)$ giving a final $1: 20(\mathrm{v} / \mathrm{v})$ dilution ratio. Duplicate samples were prepared for further processing. Acidified ethanol in a final 1:10 (v/v) dilution ratio was added to the tissue samples and homogenized using a Polytron ${ }^{\circledR}$ Benchtop Homogenizer. The digested blood and tissue samples were incubated for $24 \mathrm{~h}$ at $4^{\circ} \mathrm{C}$ in the dark. Following incubation, the precipitate was removed by centrifugation $\left(20,000 \mathrm{~g}, 20 \mathrm{~min}, 4^{\circ} \mathrm{C}\right)$ and the supernatant (containing extracted DXR) stored at $-20^{\circ} \mathrm{C}$ until fluorescence measurements were performed. The extracted DXR was quantified by fluorescence measurements as previously described using multipoint calibration curves. The latter were generated by adding known amounts of Caelyx ${ }^{\circledR}$ to blood and tissue (tumor, liver, spleen and kidney) homogenate and incubated and centrifuged as described above. All calibration curves had regression coefficients $\left(R^{2}\right)>0.99$. By adding known amounts of liposomal DXR to blood and tissue homogenate, the assay had a recovery greater than $91 \%$ for all studied tissues.

\section{Therapy study}

Animals carrying CWR22 xenografts were randomly allocated into four groups $(n=8)$ according to different treatment regimes (Table 1). A dose of $3.5 \mathrm{mg} / \mathrm{kg}$ liposomal DXR was given i.v. to anesthetized animals. The rationale for using the relatively low dose of $3.5 \mathrm{mg} / \mathrm{kg}$ liposomal DXR was to avoid reaching therapy saturation levels where any potential effects produced by US would not be detectable. Twenty-four hours postinjection, when the tumor DXR concentration had reached peak levels (see "Pharmacokinetic and biodistribution studies" section), designated animals received LFUS treatment. In brief, a $40 \mathrm{kHz}$ ultrasonic processor (model VC 754; Sonic and Materials Inc.) with a $19 \mathrm{~mm}$ diameter probe was partially submerged into a cylinder containing deionized water, degassed by boiling, and cooled in ice bath. The bottom of the cylinder was sealed with a latex membrane in firm contact with the skin covering the tumor of the 
anesthetized animal located on an adjustable plate. A thin layer of US gel was placed between the skin and the latex membrane. The US probe was run at a nominal intensity of $\sim 12 \mathrm{~W} / \mathrm{cm}^{2}$ for a duration of $4 \mathrm{~min}$ and with a $2 \mathrm{~cm}$ distance between the probe and skin. Preliminary tests were performed to ensure that the combination of probe-skin distance and duration of US exposure did not induce any visible local skin lesions. Tumor size was measured in 3-5-day intervals for 22 days using digital callipers (model B220S; Kroeplin, Schlüchtern, Germany). Tumor volume was calculated using the formula $(\pi / 6) \times$ length $^{2} \times$ width (Favier et al., 2007). Individual tumor volumes were normalized to pre-treatment level on day 0 by dividing all tumor volumes with measured volume at day 0 .

\section{Statistical analysis}

By means of a multiple regression procedure, the differences in tumor growth delay between the four experimental groups were operationally represented by three between-group contrasts: (i) comparing the liposomal DXR groups and the saline groups, (ii) comparing the LFUS group with no-LFUS within the liposomal DXR conditions and finally (iii) comparing the LFUS group with no-LFUS within the saline conditions. The analysis was performed using normalized tumor volumes. The tumor growth delay was represented by developmental growth curves of linear and quadratic polynomial contrasts, respectively, adjusted for unequal time intervals between the measurement points (Pearson and Hartley, 1976; Cohen et al., 2003).

\section{Results}

\section{In vitro liposomal characterization}

Small-sized liposomes, slightly below $100 \mathrm{~nm}$, are preferred to obtain long circulation in blood and consequently high extent of accumulation in tissues of increased vascular permeability, such as tumors (Drummond et al., 1999). The liposomes showed a mean diameter of $85 \pm 2 \mathrm{~nm}$. The mean PI of $0.077 \pm 0$ indicated a narrow size distribution. The measured $\zeta$ potential was $-19.5 \pm 1.2 \mathrm{mV}$. This slightly negative $\zeta$ potential is typical for pegylated liposomes (Woodle et al., 1992, 1994).

US-mediated DXR release from liposomes are shown in Figure 1. Release extent increased with US exposure time and reached $25 \%$ after $6 \mathrm{~min}$.

Accumulation of liposomes in tumors is a relatively slow process requiring long circulation time (Gabizon et al., 1997) and it is therefore important that circulating liposomes efficiently retain DXR en route to target. Stability studies of the investigated liposomal formulation in $20 \%$ serum demonstrated only $2 \%$ DXR leakage after 24-h incubation at $37^{\circ} \mathrm{C}$, suggesting that the liposomes would efficiently retain DXR after i.v. injection.

The liposomes also showed to be thermostable as no detectable leakage of entrapped DXR was observed during 2 -h exposure to $60^{\circ} \mathrm{C}$. The sample temperature during an US run never exceeded $30^{\circ} \mathrm{C}$, indicating that the observed in vitro drug release was not directly attributable to temperature effects.

\section{Pharmacokinetic and biodistribution studies}

Analysis of blood samples showed that $\sim 6.5 \mu \mathrm{g} / \mathrm{ml}$ of injected DXR remained in the blood circulation $24 \mathrm{~h}$ after i.v. injection (Figure 2) corresponding to $\sim 10 \%$ of the injected dose. The tumor DXR concentration reached a plateau between 24 and $48 \mathrm{~h}$ postinjection (Figure 3 ). As expected, high amounts of DXR were also found in spleen (Figure 4) and liver (Figure 5) with peak levels $24 \mathrm{~h}$ postinjection of, respectively, 8 and $35 \%$ of the injected dose, corresponding to 66 and $21 \mu \mathrm{g}$ DXR/g tissue. In the kidneys, peak DXR concentration occurred $3 \mathrm{~h}$ postinjection followed by a gradual decrease (Figure 6).

\section{Therapy study}

Tumors were exposed to LFUS $24 \mathrm{~h}$ postliposome injection coinciding with the peak DXR tumor concentration as determined in the biodistribution study. No difference in

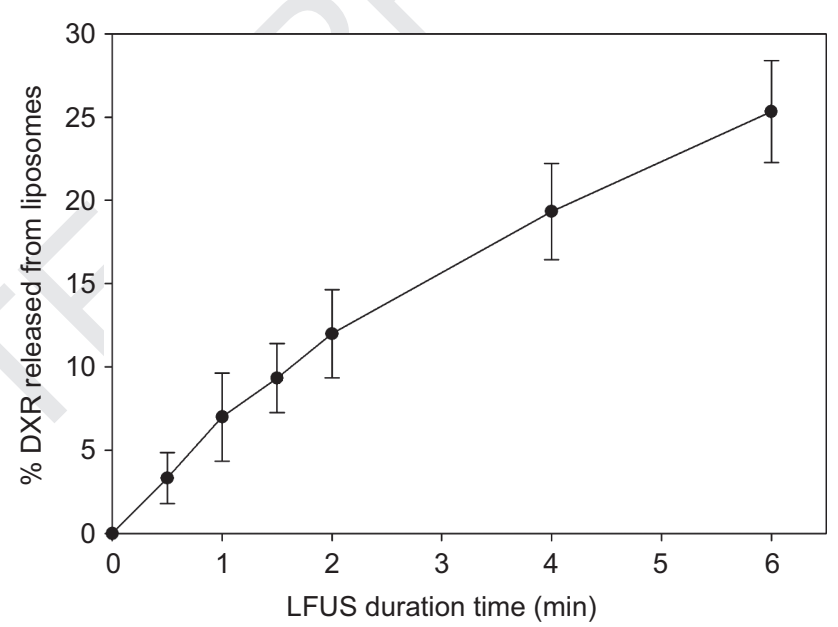

Figure 1. In vitro US-mediated DXR release from liposomes in isosmotic sucrose solution ( $40 \mathrm{kHz}$, continuous mode). The mean and standard deviation of triplicate measurements are given.

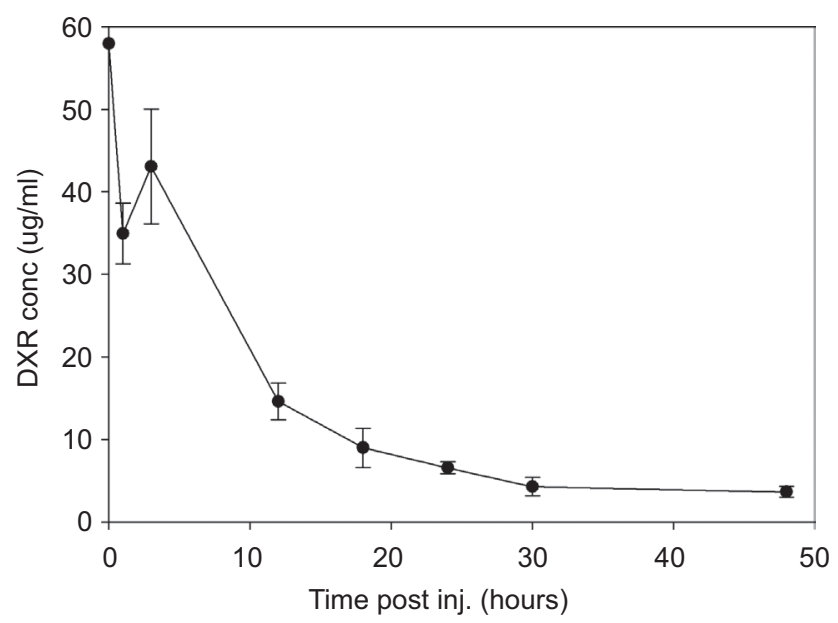

Figure 2. DXR blood concentration as a function of time after i.v. injection of liposomal DXR to tumor-bearing mice. The mean and standard deviation are given $(n=4)$.

\section{9}




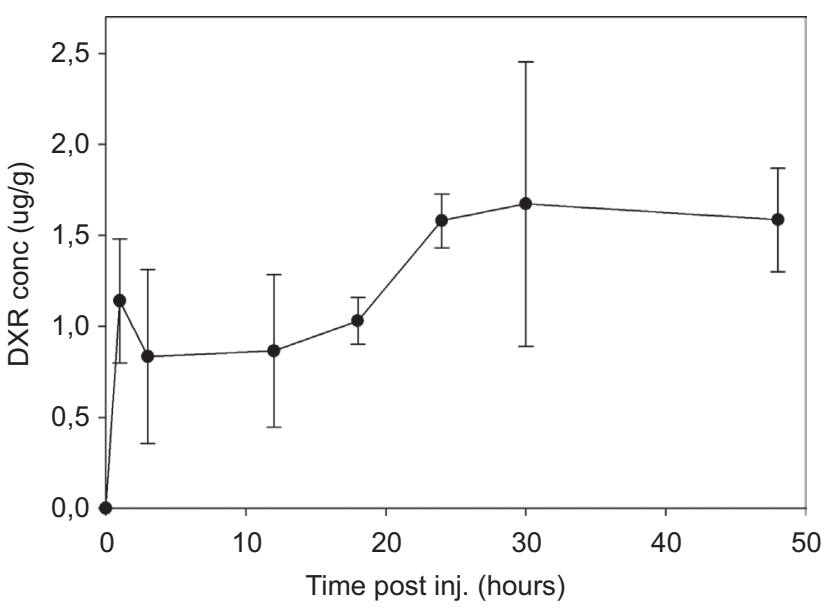

Figure 3. DXR tumor concentration as a function of time after i.v. injection of liposomal DXR to tumor-bearing mice. The mean and standard deviation are given $(n=4)$.

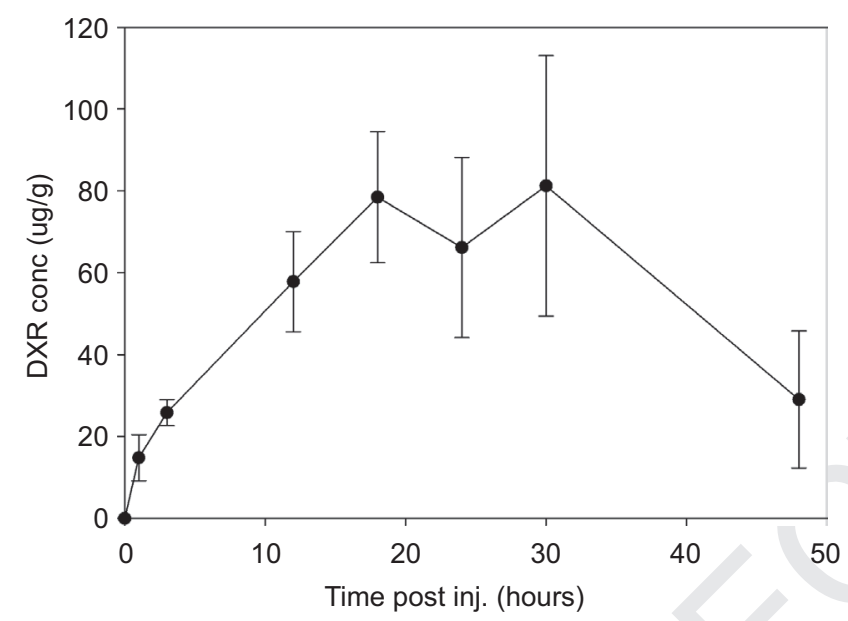

Figure 4. DXR spleen concentration as a function of time after i.v. injection of liposomal DXR to tumor-bearing mice. The mean and standard deviation are given $(n=4)$.

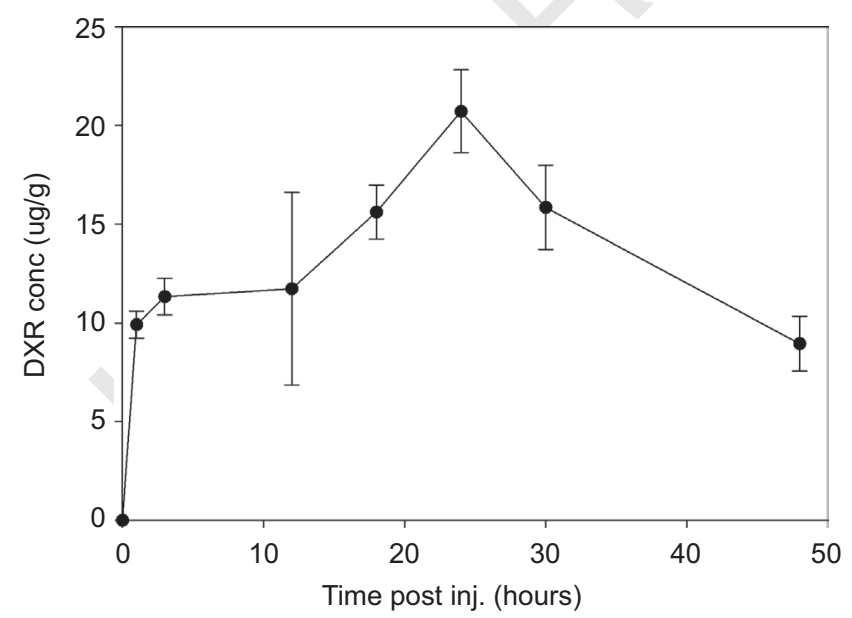

Figure 5. DXR liver concentration as a function of time after i.v. injection of liposomal DXR to tumor-bearing mice. The mean and standard deviation are given $(n=4)$.

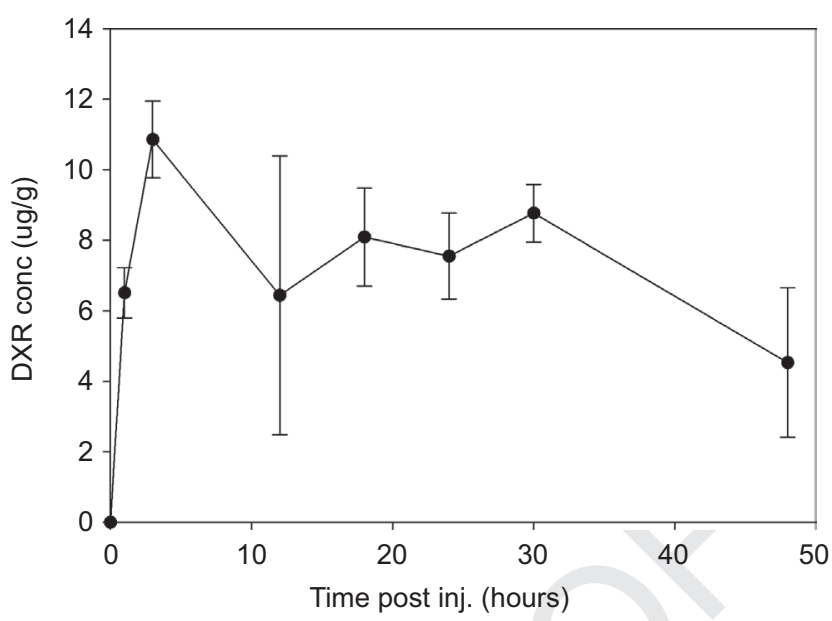

Figure 6. DXR concentration in kidneys as a function of time after i.v. injection of liposomal DXR to tumor-bearing mice. The mean and standard deviation are given $(n=4)$.

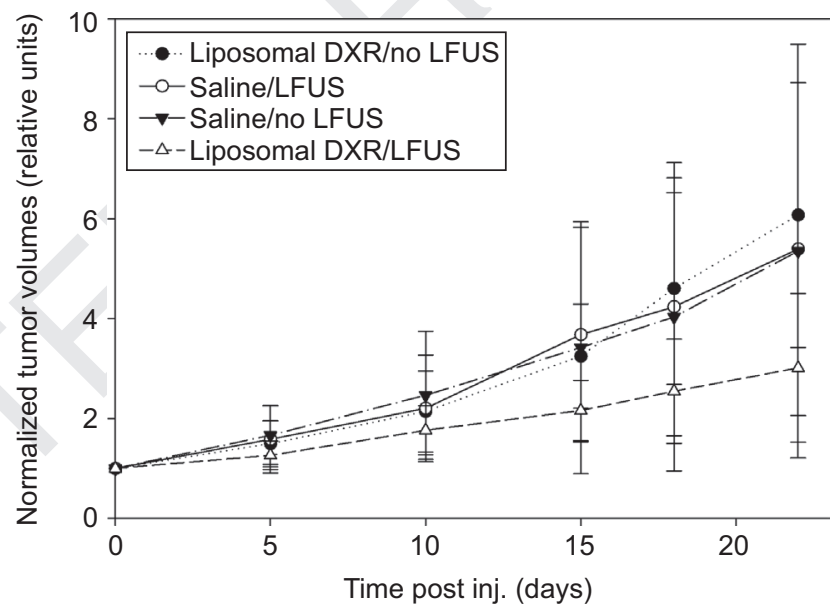

Figure 7. Tumor growth in mice receiving different treatments (Table 1). Mean and standard deviations are given $(n=8)$.

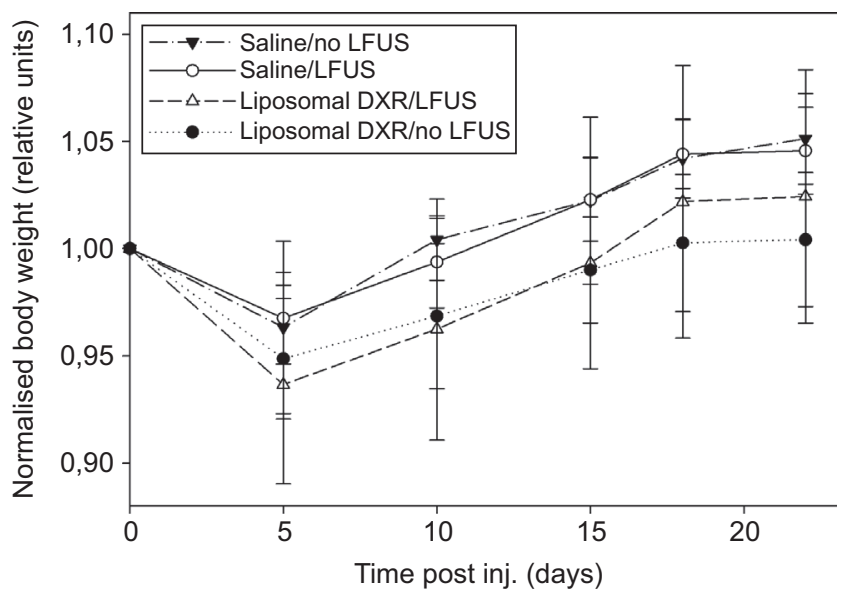

Figure 8. Normalized body weight time evolution of mice receiving different treatments (Table 1). Mean and standard deviation are given $(n=8)$.

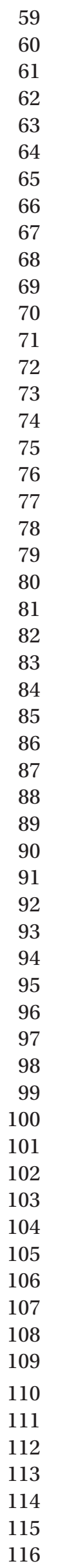


the linear trend for tumor growth was obtained between the liposomal DXR groups and saline groups (contrast 1, "Statistical analysis" section). Also for the difference between the LFUS vs. no-LFUS groups within the saline conditions, no effect was proved significant (contrast 3, "Statistical analysis" section).

However, mice receiving liposomal DXR in combination with LFUS demonstrated a significantly slower linear growth trend compared to those receiving only liposomal DXR (contrast 2, "Statistical analysis" section), controlled for the two other contrasts $(p<0.5)$. No relationships between any of the contrasts and the quadratic trend controlled for the linear trend were obtained. The differential observed pattern of growth curves for the four experimental conditions is portrayed in Figure 7.

\section{Preliminary toxicity}

The mice were monitored throughout the therapy study with respect to potential weight loss (Figure 8) and other external signs indicative of toxicity. After drug administration under anesthesia, all experimental groups experienced an initial weight loss independent of treatment. The two groups receiving liposomal DXR experienced a larger weight loss, but the mean maximum weight reduction was less than $10 \%$ of initial body weight and less than $5 \%$ of untreated control (group 3), which was considered acceptable. US treatment per se did not produce any visible skin lesions. No animals succumbed or showed signs of discomfort during the 22-day-long experiment.

\section{Discussion}

Cytostatic drugs used in the treatment of solid tumors have a non-specific distribution resulting in numerous off-target effects and suboptimal therapeutic-to-toxicity ratio. Although liposomal formulations of cytostatic drugs like DXR reduce side effects and improve tumor accumulation (Gabizon et al., 2003), efficient release from liposomes in the tumor is still considered a challenge. This challenge has been sought accommodated by triggered liposomal release with LFUS (Frenkel, 2008; Schroeder et al., 2009b).

To obtain maximum effect of LFUS treatment, it is desired to expose the tumor to LFUS at the time of maximum DXR concentration. Numerous biodistribution studies performed in tumored mice with different PL-DXR formulations and drug dosages all describe a peak DXR tumor concentration 1-2 days postinjection (Gabizon et al., 1997, 2003; Cui et al., 2007). This was confirmed in the present study and LFUS treatment was consequently performed $24 \mathrm{~h}$ postinjection. The method used for quantification of tumor DXR concentrations did not differentiate between free DXR and liposomal DXR nor between extravasated and circulating liposomal DXR in tumor tissue. However, since the blood concentration of DXR at the point of LFUS treatment (24h postinjection) represented only $10 \%$ of the injected dose, the contribution of DXR in the tumor vasculature to the total tumor DXR concentration was expected to be minimal. It follows that most of the measured DXR in tumor tissue may be assumed to be interstitial rather than circulating liposomal DXR.

Further, we show improved therapeutic outcome in tumor-bearing mice receiving liposomal DXR with subsequent exposure to LFUS. By contrast, neither liposomal DXR nor LFUS alone produced any therapeutic effect compared to untreated control. In vitro studies showed an $\sim 20 \%$ DXR release from liposomes after 4 min of US exposure and it may be assumed that similar release levels are obtained in tumor tissue. This assumption is, however, not unproblematic. Acoustic cavitation has been hypothesized as a key factor in US-mediated drug release (Pitt et al., 2004) and it is known that the threshold for inducing cavitation in a medium depends on several factors like the level of dissolved gases, viscosity, purity, etc (Atchley et al., 1988; Deng et al., 1996; Ogawa et al., 2002). These factors clearly vary between the current experimental systems rendering the above assumption uncertain. Furthermore, the liposome sonosensitivity may be directly influenced by interaction with plasma proteins, cells, etc within the tumor tissue. It is therefore possible that the observed tumor growth inhibition resulted from other effects induced by LFUS, in particular enhanced cellular drug uptake (Sundaram et al., 2003; Pitt et al., 2004) and improved liposomal extravasation (Dvorak et al., 2004).

During the 4-min in vivo exposure, the temperature of the water inside the cylinder increased from $\sim 4$ to $42^{\circ} \mathrm{C}$. A similar temperature increase in the treated tumor cannot be excluded. In vitro release studies excluded temperature as factor in drug release and no tumor growth effects of LFUS alone could be demonstrated. It follows that if intratumor temperature increased, it did not induce drug release nor was it tumouricidic alone.

All the above theories may alone or in combination explain the observed therapeutic effect of liposomal DXR combined with LFUS treatment. Surprisingly, the group receiving solely liposomal DXR did not experience any anti-tumor effect suggesting that tumoricidic concentrations of free DXR in the tumor were not obtained. The in vitro serum stability studies and in vivo pharmacokinetic studies demonstrated high stability and reduced bioavailability of the encapsulated DXR. This may both explain why tumouricidic levels of free DXR was not obtained in the group receiving solely liposomal DXR, as well as provide support for LFUStriggered drug release in the group receiving liposomal DXR in combination with LFUS. Still, a conclusion on the mechanism of action will have to await further studies distinguishing between free and liposomal DXR post-LFUS treatment.

There were relatively large variations in measured tumor DXR concentrations between mice within the same groups. This heterogeneous liposome uptake is likely to 
Table 1. Experimental groups receiving different treatments.

\begin{tabular}{|c|c|c|c|}
\hline \multicolumn{4}{|c|}{ Experimental groups } \\
\hline Group no & $n$ & Administered test article & LFUS \\
\hline 1 & 8 & Liposomal DXR (3.5 mg/kg) & - \\
\hline 2 & 8 & Liposomal DXR ( $3.5 \mathrm{mg} / \mathrm{kg})$ & + \\
\hline 3 & 8 & Saline (0.25 ml/animal) & - \\
\hline 4 & 8 & Saline (0.25 ml/animal) & + \\
\hline
\end{tabular}

be caused by intratumoural differences in physiological features such as necrosis and vascularization. Also, the CWR22 tumor model is an androgen-dependent human prostate adenocarcinoma and individual differences in tumor growth rate may, to a certain extent, be explained by differences in androgen levels between individual animals.

Acute toxicity studies with PL-DXR in mice have reported $\mathrm{LD}_{10}$ and $\mathrm{LD}_{50}$ to be $11.7 \pm 2.1$ and $38.3 \pm 7.2 \mathrm{mg}$ $\mathrm{DXR} / \mathrm{kg}$, respectively (Working and Dayan, 1996). Accumulated doses of $36 \mathrm{mg}$ DXR/ $\mathrm{kg}$ of PL-DXR have also reported to give severe toxicities in mice (Charrois and Allen, 2003). The current liposomal DXR dose of $3.5 \mathrm{mg} / \mathrm{kg}$ is well below these levels and no adverse effects were observed. The biodistribution study revealed substantial liver uptake and liver toxicities could be a potential problem. However, in this study, only moderate therapy associated weight loss was observed during the first days postdrug administration and did not deviate significantly from untreated control. Hence, the probable cause of weight loss was anesthesia rather than toxic effects of the liposomal DXR formulation. Animals receiving LFUS did not develop any local skin lesions nor did they experience any additional weight loss.

\section{Conclusion}

In this study, a new treatment strategy combining DSPEbased liposomal DXR and LFUS in tumor-bearing mice has been demonstrated. The data suggest an enhanced antitumor effect of liposomal DXR when combined with acoustic energy as observed by a significant tumor growth delay. However, as US may have complex effects on exposed tissues, new studies are necessary to clarify the mechanism behind the observed therapeutic effect. Also, optimization of liposome sonosensitivity at clinically relevant US frequencies will be important upon clinical implementation.

\section{Acknowledgements}

The project is supported by the Nanomat programme, the Norwegian Research Council. We thank Professor Knut A. Hagtvet, University of Oslo, for valuable advice regarding statistical analysis, as well as Sibylla Røgnvaldsson and Ulrich M. W. Eide for technical assistance. We also like to thank Professor F. Saatcioglu, University of Oslo, for providing the CWR22 xenograft model.

\section{Declaration of interest}

TJE, SLF, EAN and Sibylla Røgnvaldsson have ownership interests in Epitarget AS. EH and DRO have no such interests in Epitarget AS and report no conflict of interest. The authors alone are responsible for the content and writing of the manuscript, and the decision to publish.

\section{References}

Allen TM, Cleland LG. (1980). Serum-induced leakage of liposome contents. Biochim Biophys Acta, 597, 418-426.

Atchley AA, Frizzell LA, Apfel RE, Holland CK, Madanshetty S, Roy RA. (1988). Thresholds for cavitation produced in water by pulsed ultrasound. Ultrasonics, 26, 280-285.

Batist G. (2007). Cardiac safety of liposomal anthracyclines. Cardiovasc Toxicol, 7, 72-74.

Bonté F, Juliano RL. (1986). Interactions of liposomes with serum proteins. Chem Phys Lipids, 40, 359-372.

Charrois GJ, Allen TM. (2003). Multiple injections of pegylated liposomal Doxorubicin: pharmacokinetics and therapeutic activity. J Pharmacol Exp Ther, 306, 1058-1067.

Cohen J, Cohen P, West SG, Aiken LS. (2003). Applied multiple regression/correlation analysis for the behavioral sciences (3rd ed.) Mahwah, NJ: Erlbaum.

Cui J, Li C, Guo W, Li Y, Wang C, Zhang L, Zhang L, Hao Y, Wang Y. (2007). Direct comparison of two pegylated liposomal doxorubicin formulations: is AUC predictive for toxicity and efficacy? J Control Release, 118, 204-215.

Davidsen J, Vermehren C, Frokjaer S, Mouritsen OG, Jørgensen K. (2001). Drug delivery by phospholipase A(2) degradable liposomes. Int J Pharm, 214, 67-69.

Deng CX, Xu Q, Apfel RE, Holland CK. (1996). In vitro measurements of inertial cavitation thresholds in human blood. Ultrasound Med Biol, 22, 939-948.

Drummond DC, Meyer O, Hong K, Kirpotin DB, Papahadjopoulos D. (1999). Optimizing liposomes for delivery of chemotherapeutic agents to solid tumors. Pharmacol Rev, 51, 691-743.

Dvorak J, Zoul Z, Melichar B, Petera J, Vesely P, Vosmik M, Dolezel M. (2004). Liposomal doxorubicin combined with regional hyperthermia: reducing systemic toxicity and improving locoregional efficacy in the treatment of solid tumors. J Chemother, 16 Suppl 5, 34-36.

Evjen TJ, Nilssen EA, Rögnvaldsson S, Brandl M, Fossheim SL. (2010). Dis tearoylphosphatidylethanolamine-based liposomes for ultrasoundmediated drug delivery. Eur J Pharm Biopharm, 75, 327-333.

Ewer MS, Martin FJ, Henderson C, Shapiro CL, Benjamin RS, Gabizon AA. (2004). Cardiac safety of liposomal anthracyclines. Semin Oncol, 31, 161-181.

Favier J, Lapointe S, Maliba R, Sirois MG. (2007). HIF2 alpha reduces growth rate but promotes angiogenesis in a mouse model of neuroblastoma. BMC Cancer, 7, 139.

Frenkel V. (2008). Ultrasound mediated delivery of drugs and genes to solid tumors. Adv Drug Deliv Rev, 60, 1193-1208.

Gabizon A, Goren D, Horowitz AT, Tzemach D, Lossos A, Siegal T. (1997). Long-circulating liposomes for drug delivery in cancer therapy: a review of biodistribution studies in tumor-bearing animals. Adv Drug Deliv Rev, 24:337-44.

Gabizon A, Shiota R, Papahadjopoulos D. (1989). Pharmacokinetics and tissue distribution of doxorubicin encapsulated in stable liposomes with long circulation times. J Natl Cancer Inst, 81, 1484-1488.

Gabizon A, Shmeeda H, Barenholz Y. (2003). Pharmacokinetics of pegylated liposomal Doxorubicin: review of animal and human studies. Clin Pharmacokinet, 42, 419-436.

Huang SL, MacDonald RC. (2004). Acoustically active liposomes for drug encapsulation and ultrasound-triggered release. Biochim Biophys Acta, 1665, 134-141. 


\section{E. Hagtvet et al.}

Jang SH, Wientjes MG, Lu D, Au JL. (2003). Drug delivery and transport to solid tumors. Pharm Res, 20, 1337-1350.

Lasic DD. (1993). Liposomes from Physics to Applications. Amsterdam: Elsevier Science Publishers B.V.

Maeda H, Wu J, Sawa T, Matsumura Y, Hori K. (2000). Tumor vascular permeability and the EPR effect in macromolecular therapeutics: a review. J Control Release, 65, 271-284.

Myhr G, Moan J. (2006). Synergistic and tumour selective effects of chemotherapy and ultrasound treatment. Cancer Lett, 232, 206-213.

Needham D, Dewhirst MW. (2001). The development and testing of a new temperature-sensitive drug delivery system for the treatment of solid tumors. Adv Drug Deliv Rev, 53, 285-305.

Ogawa R, Kondo T, Honda H, Zhao QL, Fukuda S, Riesz P. (2002). Effects of dissolved gases and an echo contrast agent on ultrasound mediated in vitro gene transfection. Ultrason Sonochem, 9, 197-203.

Pearson ES, Hartley HOE. (1976). Biometrika Tables for Statisticians (vol 1). Biometrica Trust, University College London.

Pitt WG, Husseini GA, Staples BJ. (2004). Ultrasonic drug delivery-a general review. Expert Opin Drug Deliv, 1, 37-56.

Pretlow TG, Wolman SR, Micale MA, Pelley RJ, Kursh ED, Resnick MI, Bodner DR, Jacobberger JW, Delmoro CM, Giaconia JM. (1993). Xenografts of primary human prostatic carcinoma. J Natl Cancer Inst, 85, 394-398.
Schroeder A, Avnir Y, Weisman S, Najajreh Y, Gabizon A, Talmon Y, Kost J, Barenholz Y. (2007). Controlling liposomal drug release with low frequency ultrasound: mechanism and feasibility. Langmuir, $23,4019-4025$.

Schroeder A, Honen R, Turjeman K, Gabizon A, Kost J, Barenholz Y. (2009a). Ultrasound triggered release of cisplatin from liposomes in murine tumors. J Control Release, 137, 63-68.

Schroeder A, Kost J, Barenholz Y. (2009b). Ultrasound, liposomes, and drug delivery: principles for using ultrasound to control the release of drugs from liposomes. Chem Phys Lipids, 162, 1-16.

Sudimack JJ, Guo W, Tjarks W, Lee RJ. (2002). A novel pH-sensitive liposome formulation containing oleyl alcohol. Biochim Biophys Acta, 1564, 31-37.

Sundaram J, Mellein BR, Mitragotri S. (2003). An experimental and theoretical analysis of ultrasound-induced permeabilization of cell membranes. Biophys J, 84, 3087-3101.

Woodle MC, Collins LR, Sponsler E, Kossovsky N, Papahadjopoulos D, Martin FJ. (1992). Sterically stabilized liposomes. Reduction in electrophoretic mobility but not electrostatic surface potential. Biophys J, 61, 902-910.

Woodle MC, Newman MS, Cohen JA. (1994). Sterically stabilized liposomes: physical and biological properties. J Drug Target, 2, 397-403.

Working PK, Dayan AD. (1996). Pharmacological-toxicological expert report. CAELYX. (Stealth liposomal doxorubicin HCl). Hum Exp Toxicol, 15, 751-785. 